Proceedings

\title{
Porous Silicon Carbide for MEMS ${ }^{\dagger}$
}

\author{
Markus Leitgeb *, Christopher Zellner, Georg Pfusterschmied, Michael Schneider and \\ Ulrich Schmid \\ Institute of Sensor and Actuator Systems, TU Wien, Gusshausstrasse 27-29, 1040 Vienna, Austria; \\ Christopher.Zellner@tuwien.ac.at (C.Z.); georg.pfusterschmied@tuwien.ac.at (G.P.); \\ Michael.Schneider@tuwien.ac.at (M.S.); ulrich.e366.schmid@tuwien.ac.at (U.S.) \\ * Correspondence: markus.leitgeb@tuwien.ac.at; Tel.: +43-(0)1-58801-76638 \\ + Presented at the Eurosensors 2017 Conference, Paris, France, 3-6 September 2017.
}

Published: 30 August 2017

\begin{abstract}
Metal assisted photochemical etching (MAPCE) of $4 \mathrm{H}$ Silicon Carbide ( $\mathrm{SiC}$ ) was utilized to generate locally defined porous areas on single crystalline substrates. Therefore, Platinum (Pt) was sputter deposited on $4 \mathrm{H}-\mathrm{SiC}$ substrates and patterned with photolithography and lift off. Etching was performed by immersing the Pt coated samples into an etching solution containing sodium persulphate and hydrofluoric acid. UV light irradiation was necessary for charge carrier generation while the Pt served as local cathode. The generated porous areas can be used for the generation of integrated cavities in the single crystalline $\mathrm{SiC}$ substrates when covered with a chemical vapor deposited thin film of poly-crystalline SiC.
\end{abstract}

Keywords: metal assisted etching; Silicon Carbide; chemical vapor deposition

\section{Introduction}

Porous semiconductors are a popular topic in research and development, with porous silicon (pSi) as the most prominent representative. When $\mathrm{pSi}$ is annealed at temperatures up to $1100{ }^{\circ} \mathrm{C}$ it reorganizes to minimize surface energy. This effect is utilized in the fabrication of pressure sensors by the formation of cavities after high temperature annealing [1]. Furthermore, the separation of thin single crystalline silicon layers is possible [2]. Because of the chemical inertness, mechanical stability and high band gap of SiC, MEMS devices based on this wide band gap semiconductor can be operated at higher temperatures and in more aggressive environments than devices fabricated in standard silicon micromachining. Furthermore, thin SiC layers are most beneficial for UV detectors. For the realization of novel device concepts, the tailored porosification in selected areas of monocrystalline SiC substrates is regarded as a key technology.

Porous $\mathrm{SiC}(\mathrm{pSiC}$ ) can be prepared by metal assisted photochemical etching (MAPCE), which in contrast to conventional anodization of $\mathrm{SiC}$, is capable of producing uniform porous layers. In this approach a noble metal like Platinum $(\mathrm{Pt})$ is deposited on the $\mathrm{SiC}$ surface, serving as local cathode. When the sample is immersed in an etching solution containing an oxidizing agent and hydrofluoric acid, a charge transfer occurs with electrons flowing from the $\mathrm{SiC}$ substrate via the $\mathrm{Pt} / \mathrm{SiC}$ interface to the etching solution. Simultaneously $\mathrm{pSiC}$ forms at the bare $\mathrm{SiC}$ surface. Recent experiments showed that reliable etching can be achieved by decreasing the contact resistance at the $\mathrm{Pt} / \mathrm{SiC}$ junction [3].

In this paper MAPCE is utilized to generate locally defined pSiC which is necessary for the realization of device concepts mentioned above.

\section{Materials and Methods}

For the experiments square sized samples $\left(1 \times 1 \mathrm{~cm}^{2}\right)$ were cut from single crystalline $4 \mathrm{H}-\mathrm{SiC}$ wafers with different resistivities of 0.02 and $0.106 \Omega \cdot \mathrm{cm}$. Then the samples were cleaned 
consecutively with acetone and ethanol. The samples having a resistivity of $0.106 \Omega \cdot \mathrm{cm}$ were surface near doped with a diffusion based approach utilizing a phosphoric acid solution and high temperatures up to $1100{ }^{\circ} \mathrm{C}$. This was done to decrease the contact resistance during etching at the $\mathrm{Pt} / \mathrm{SiC}$ interface. The experimental details can be found elsewhere [3]. After cleaning, $10 \%$ of the sample's surface were covered with $300 \mathrm{~nm}$ Pt by sputter deposition. Next the samples were annealed for 5 min under Argon flow at $1100{ }^{\circ} \mathrm{C}$ with a prior temperature ramp starting at $800{ }^{\circ} \mathrm{C}$ that lasted for $30 \mathrm{~min}$. This was done to further decrease the contact resistance at the Pt/SiC interface. [3] After this annealing step, $100 \mathrm{~nm}$ Pt were again sputter deposited and structured with photolithography and lift off. This was done because the annealing and doping processes lead to surface roughening. By the chosen two-step process, structuring of the samples could be done at smooth parts of the samples. Finally the samples were immersed in an etching solution containing $0.04 \mathrm{~mol} / \mathrm{L}$ sodium persulphate and $1.31 \mathrm{~mol} / \mathrm{L}$ hydrofluoric acid (HF). In the case of samples with a resistivity of $0.02 \Omega \cdot \mathrm{cm}$ $1.2 \mathrm{~mL}$ of etching solution were used while a custom built $18 \mathrm{~W}$ UVC source was used for irradiation. For samples with higher resistivity, $150 \mathrm{~mL}$ of etching solution were used while a 250 Watt ES280LL mercury arc lamp at full spectrum was applied for irradiation. After etching for $2 \mathrm{~h}$ the Pt was removed with boiling aqua regia and polycrystalline $\mathrm{SiC}$ was deposited with a hot wall low pressure chemical vapor deposition (LPCVD) furnace from FirstNano ${ }^{\circledast}$. The $\mathrm{SiC}$ films were deposited at $1050^{\circ} \mathrm{C}$ using gas flows of $1 \mathrm{sccm} \mathrm{SiH}$ and $60 \mathrm{sccm} \mathrm{CH}_{4}$ with $500 \mathrm{sccm} \mathrm{H}_{2}$ as background gas.

\section{Discussion}

During etching porous layers were generated at the bare SiC surface which had not been covered with Pt. This is illustrated in Figure 1. One can see a secondary electron microscope (SEM) micrograph showing pSiC and the schematically indicated charge carrier flow paths during etching. UV light generates charge carries (electrons $\mathrm{e}^{-}$, holes $\mathrm{h}^{+}$). The holes, together with $\mathrm{H}_{2} \mathrm{O}$ are responsible for the oxidation of $\mathrm{SiC}$, while the oxide is afterwards dissolved by $\mathrm{HF}$ leaving behind $\mathrm{pSiC}$. To restore charge neutrality during etching, the electrons are accepted from the oxidizing agent $\left(\mathrm{SO}_{4}{ }^{-}\right)$at the Pt/etching solution interface [3].

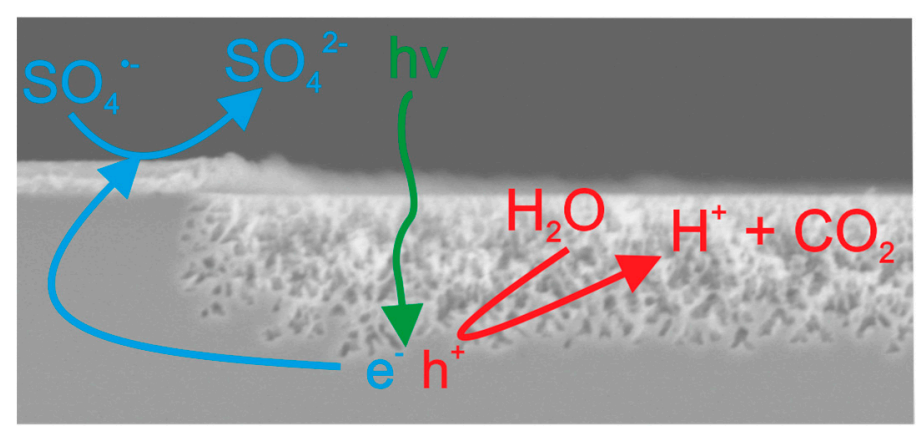

Figure 1. Schematic depiction of the mechanism of MAPCE.

For successful application of the generated $\mathrm{pSiC}$ layers, the Pt cathodes have to be removed completely after MAPCE without damaging or dissolving the pSiC. This was possible with aqua regia, as is shown in Figure 2 (sample resistivity of $0.106 \Omega \cdot \mathrm{cm}$ ). One can see that the $\mathrm{pSiC}$ remains intact after removing the $\mathrm{Pt}$ with boiling aqua regia (see Figure 2a). A corresponding top down view (see Figure $2 b$ ) shows that it is possible to locally define pSiC on single crystalline substrates. The orange areas show $\mathrm{pSiC}$ which is surrounded by dense $\mathrm{SiC}$.

Many possible application scenarios, such as the layer transfer method or epitaxial growth of GaN [4], require the deposition of thin films via CVD without filling the porous material. This could in principle be shown with a sample having a resistivity of $0.106 \Omega \cdot \mathrm{cm}$, as is illustrated in Figure 3. It can be seen that a pSiC area is covered with polycrystalline $\mathrm{SiC}$, while the porous layer was not refilled with polycrystalline SiC. 


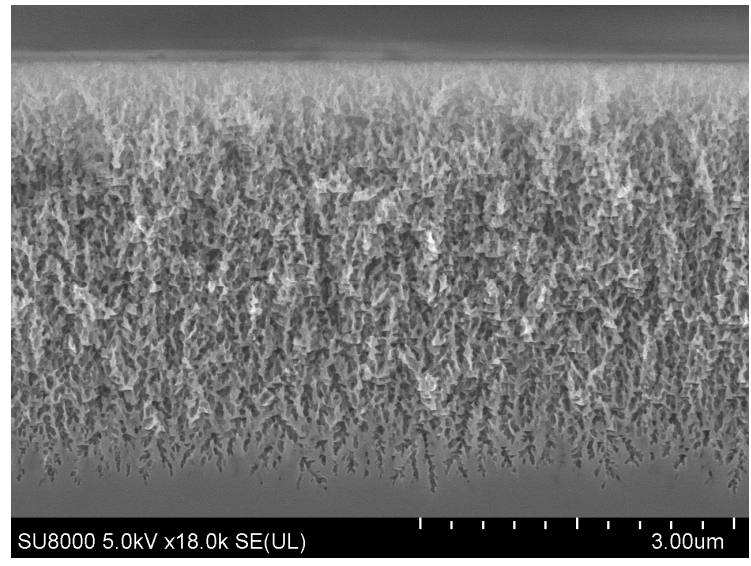

(a)



(b)

Figure 2. Porous SiC generated with MAPCE after Pt removal with aqua regia; (a) Cross sectional SEM micrograph; (b) Top down view optical microscope image. The orange areas indicate regions of pSiC surrounded by dense SiC.

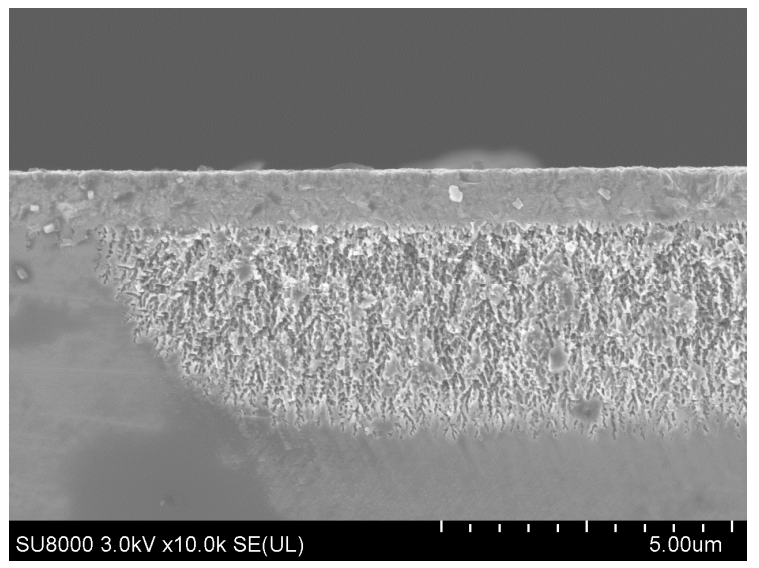

Figure 3. Porous silicon carbide covered with a polycrystalline SiC layer deposited with LPCVD.

Another possible application scenario of the process described so far is the formation of cavities for e.g., pressure sensor fabrication as demonstrated by Armbruster et al. for pSi [1]. In this approach pSi reorganizes under high temperatures leaving behind a cavity covered with a membrane on top. This membrane is used for pressure sensor applications.

This approach is also possible for $\mathrm{SiC}$ substrates by utilizing MAPCE. Samples annealed at $1500{ }^{\circ} \mathrm{C}$ in air showed reorganization of the $\mathrm{pSiC}$ as is shown in Figure 4. A cavity has formed with polycrystalline $\mathrm{SiC}$ on top and single crystalline $4 \mathrm{H} \mathrm{SiC}$ at the bottom. This sample had a resistivity of $0.02 \Omega \cdot \mathrm{cm}$. Besides the successful reorganization of pSiC it was also possible to use a low power UV source and a small amount of etchant for samples with lower resistivities (see experimental details). This is explained with a lower resistance for electron transport through the bulk $\mathrm{SiC}$ material during etching and is of economical as well as practical importance when toxic etching solutions are used.

In summary it can be stated that $\mathrm{pSiC}$ can be reliably generated with MAPCE and the presented results demonstrate the potential of this process for the realization of SiC-based MEMS/NEMS devices. 


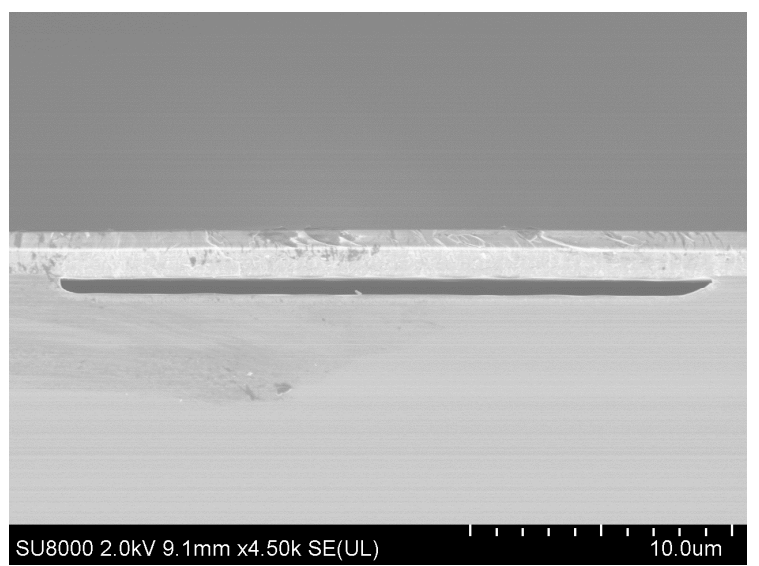

Figure 4. A micromachined cavity with bulk $4 \mathrm{H}-\mathrm{SiC}$ at the bottom and polycrystalline $\mathrm{SiC}$ on top. As the annealing step was performed in air an oxide layers has formed on the poly-SiC membrane.

Acknowledgments: This project has been supported by the COMET K1 centre ASSIC Austrian Smart Systems Integration Research Center. The COMET-Competence Centers for Excellent Technologies-Programme is supported by BMVIT, BMWFW and the federal provinces of Carinthia and Styria.

Conflicts of Interest: The authors declare no conflict of interest.

\section{References}

1. Armbruster, S.; Schafer, F.; Lammel, G.; Artmann, H.; Schelling, C.; Benzel, H.; Finkbeiner, S.; Larmer, F.; Ruther, R.; Paul, O. A novel micromachining process for the fabrication of monocrystalline Si-membranes using porous silicon. In Proceedings of the TRANSDUCERS, Solid-State Sensors, Actuators and Microsystems International Conference, 2003; pp. 246-249.

2. Terheiden, B.; Hensen, J.; Wolf, A.; Horbelt, R.; Plagwitz, H.; Brendel, R. Layer Transfer from Chemically Etched $150 \mathrm{~mm}$ Porous Si Substrates. Materials 2011, 4, 941-951.

3. Leitgeb, M.; Backes, A.; Zellner, C.; Schneider, M.; Schmid, U. Communication-The Role of the MetalSemiconductor Junction in Pt-Assisted Photochemical Etching of Silicon Carbide. ECS J. Solid State Sci. Technol. 2016, 5, P148-P150.

4. Inoki, C.K.; Kuan, T.S.; Sagar, A.; Lee, C.D.; Feenstra, R.M.; Koleske, D.D.; Diaz, D.J.; Bohn, P.W.; Adesida, I. Growth of GaN on porous SiC and GaN substrates. Phys. Status Solidi A 2003, 200, 44-47.

(C) 2017 by the authors. Licensee MDPI, Basel, Switzerland. This article is an open access article distributed under the terms and conditions of the Creative Commons Attribution (CC BY) license (http://creativecommons.org/licenses/by/4.0/). 